\title{
Potencial e qualidade da madeira de desbaste de teca para produção de biocombustível
}

\author{
Dimas Agostinho da Silva ${ }^{1}$, Eloísa Rodycz de Christo ${ }^{1}$, Alexandre Belhing ${ }^{1}$, Sandra Lucia Soares Mayer ${ }^{1 *}$, \\ Karine Andressa Pelanda ${ }^{1}$, Rodrigo Simetti ${ }^{1}$ \\ Universidade Federal do Paraná, Departamento de Engenharia e Tecnologia Florestal, Av. Pref. Lothário Meissner, 900, Jd. Botânico, CEP 80210-170, Curitiba, \\ PR, Brasil
}

\section{"Autor correspondente: \\ sandra_1smayer@yahoo.com.br}

Termos para indexação:

Tectona grandis

Propriedades energéticas

Energia de Biomassa

Index terms:

Tectona grandis

Energetic properties

Biomass Energy

Histórico do artigo:

Recebido em 07 nov 2014

Aprovado em 04 set 2015

Publicado em 30 set 2015

doi: 10.4336/2015.pfb.35.83.817

\begin{abstract}
Resumo - O presente trabalho teve como objetivo analisar o potencial energético e a qualidade da madeira de Tectona grandis Linn F. proveniente de diferentes desbastes para produção de energia. O material de estudo foi oriundo de um plantio no município de São José dos Quatro Marcos, MT. Foram realizadas as análises de poder calorífico superior, composição química imediata e densidade básica. A análise de variância não mostrou diferença ao longo do fuste para todas as propriedades estudadas. Entretanto, houve variação significativa a $1 \%$ de probabilidade para a densidade em função da idade, no qual as maiores médias foram provenientes do segundo desbaste $(0,584$ g.cm-3). As médias entre posição e idade de desbaste foram de $4.594 \mathrm{kcal} . \mathrm{kg}^{-1}$ para o poder calorífico superior; $80,37 \%$ para materiais voláteis; $18,39 \%$ para carbono fixo e $0,59 \%$ para cinzas.
\end{abstract}

\section{Potential and quality of teak wood from thinning for biofuel production}

\begin{abstract}
This study aimed to analyze the energy potential and wood quality of Tectona grandis Linn F., using different positions along the stem (base, middle, and top) and thinning ages (56 and 92 months). The study material was from the city of São José dos Quatro Marcos, MT, Brazil. Analyses of higher heating value, proximate chemical composition and density were performed. Analysis of variance revealed no differences along the stem for all properties studied. However, there was significant variation at $1 \%$ probability density as a function of age, in which the highest averages were from the second thinning $\left(0.584 \mathrm{~g} . \mathrm{cm}^{-3}\right)$. The average position between thinning and age were $4,594 \mathrm{kcal} . \mathrm{kg}^{-1}$ for higher heating value; $80.37 \%$ for volatile materials; $18.39 \%$ for fixed carbon and $0.59 \%$ for ash.
\end{abstract}

\section{Introdução}

A biomassa florestal tem sido historicamente utilizada pelo homem para a geração de energia em diversas regiões do planeta, por meio de diferentes processos de conversão energética. Entre os processos mais utilizados destacam-se a combustão direta, a gaseificação e a carbonização (Silva, 2001).

A energia de base florestal tem muitas vantagens em relação aos combustíveis fosseis: é uma fonte renovável, mais barata, menos poluente, e permite o reaproveitamento de resíduos (Silva et al., 2010). Estas características tornam a biomassa florestal uma opção importante na matriz energética não só brasileira, como mundial.

A biomassa para produção de energia pode ser oriunda de diversas fontes. De acordo com Silva et al. (2014b) o uso da madeira oriunda de plantios florestais é uma excelente opção para produção de energia e tem ganho cada vez mais destaque no cenário mundial. Nesse 
trabalho será dado ênfase para biomassa oriunda de desbastes de povoamentos de teca (Tectona grandis Linn F.), uma vez que esse tem sido um dos principais destinos dessa madeira (González, 2004; Pelissari et al., 2014).

T. grandis é uma espécie de grande porte, pertencente à família Lamiaceae (ex Verbenaceae) (Tropicos, 2015) e é nativa do continente asiático (Pandey \& Brown, 2000; Nocetti et al., 2011). As características botânicas da espécie podem ser consultadas em Pelissari et al. (2014).

A qualidade da madeira dessa espécie, voltada principalmente para usos nobres, fez com que alcançasse destaque mundial, sendo cultivada em muitos países. No Brasil, a teca foi introduzida em 1968 e os plantios concentram-se nas regiões Centro-Oeste e Norte, e o estado do Mato Grosso é que detém a maior área plantada (Shimizu et al, 2007; Schuhli \& Paludzyszyn Filho, 2010; Pelissari et al., 2014).

De acordo com Garcia (2006), no Brasil, a teca é cultivada sob espaçamento $3 \mathrm{~m} \times 2 \mathrm{~m}$, e são realizados desbastes aos 5, 10, 15 e 20 anos. Pelissari et al. (2014) indicam que a tendência é utilizar espaçamentos de $3,5 \mathrm{~m} \times 3 \mathrm{~m}$ ou $4 \mathrm{~m} \times 2,5 \mathrm{~m}$, com objetivo de facilitar as operações com máquinas agrícolas.

A produtividade média situa-se entre 10 e $15 \mathrm{~m}^{3}$.ha ${ }^{-1}$.ano ${ }^{-1}$ até os 25 anos em regime com quatro desbastes (Angeli, 2003). Pelissari et al. (2014), após revisarem diferentes trabalhos na literatura, observaram que para uma rotação de 35 a 55 anos, a produtividade varia entre 5 e $16 \mathrm{~m}^{3} \cdot \mathrm{ha}^{-1}$. no $^{-1}$, na África, e entre 10 e $20 \mathrm{~m}^{3} \cdot$ ha $^{-1}$. ano $^{-1}$ nas Américas do Sul e Central, para uma rotação de 20 a 25 anos.

Segundo Pelissari et al. (2013) a teca é afetada pela competição intraespecífica e isso exige a aplicação de desbastes em diferentes intensidades e períodos. De acordo com Pelissari et al. (2014), normalmente os povoamentos são mantidos com densidade inicial (1.000 a 2.000 árvores por hectare) até o quarto ou quinto ano após o plantio e, posteriormente, são realizados desbastes seletivos com intensidades entre $40 \%$ a $60 \%$ do número de indivíduos por hectare.

Flórez (2012) pesquisou as propriedades tecnológicas da madeira dessa espécie, e observou valores de poder calorífico superior (PCS) que variam entre $4.742 \mathrm{e}$ $4.787 \mathrm{kcal} . \mathrm{kg}^{-1}$, com média de $4.765,8 \mathrm{kcal} . \mathrm{kg}^{-1}$. O autor destaca que o PCS foi maior ao normalmente encontrado para madeira de folhosas, concluindo que é uma espécie com boas características para geração de energia.
No entanto, ainda são escassas as informações relacionadas às propriedades energéticas da madeira de $T$. grandis proveniente de desbastes, em diferentes idades. Diante do exposto, este trabalho teve por objetivo analisar o potencial energético e a qualidade da madeira de Tectona grandis proveniente de diferentes desbastes para produção de energia.

\section{Material e métodos}

Foi utilizado material proveniente de duas fazendas da Empresa Soroteca Agroflorestal, localizadas no município de São José dos Quatro Marcos, MT, latitude $15^{\circ} 37^{\prime} 17^{\prime \prime} \mathrm{S}$ e longitude $58^{\circ} 10^{\prime} 35^{\prime \prime} \mathrm{W}$.

$\mathrm{O}$ espaçamento inicial do plantio foi de $3 \mathrm{mx}$ $3 \mathrm{~m}$, sendo que o primeiro desbaste foi realizado aos 56 meses, com remoção de $35 \%$ dos indivíduos, e o segundo desbaste aos 92 meses, com remoção de 30\% dos indivíduos.

Os dados dendrométricos medidos no povoamento dos desbastes foram diâmetro a 1,30 m do solo (DAP) e altura do fuste. Também foi obtido o número de árvores desbastadas por hectare.

Foram amostradas 10 árvores por povoamento, sendo 5 oriundas do primeiro desbaste e 5 do segundo. As amostras foram coletadas na base, no meio e no topo do fuste, para cada árvore, totalizando 30 amostras.

A densidade básica da madeira foi determinada conforme a norma NBR 11941/02 (Associação Brasileira de Normas Técnicas, 2003). O poder calorífico superior determinado segundo a norma NBR 8633/84 (Associação Brasileira de Normas Técnicas, 1984) utilizando calorímetro (C5000, IKA WORKS, Germany) e a composição química imediata, para obtenção dos teores de materiais voláteis, carbono fixo e cinzas, foi determinada conforme a norma NBR 8112/86 (Associação Brasileira de Normas Técnicas, 1986). Cada análise foi realizada em duplicata para cada amostra.

Foi aplicada análise de variância para testar a hipótese da nulidade a $95 \%$ e $99 \%$ de probabilidade. A hipótese testada foi que as propriedades energéticas avaliadas se mantêm constantes ao variar a idade do povoamento ou a posição ao longo do fuste. Quando algum fator apresentou significância, foi aplicado o teste de prova de médias de Tukey a 95\% de probabilidade.

$\mathrm{O}$ volume de madeira dos desbastes foi estimado utilizando-se a Equação 1, conforme as concepções apresentadas por Loetsch et al. (1973). 


$$
V M d=\frac{D A P^{2} * \pi}{40000} * H^{*} F F^{*} N
$$

Em que:

VMd - volume de madeira de desbastes, $\mathrm{em} \mathrm{m}^{3} \cdot \mathrm{ha}^{-1}$;

DAP - diâmetro médio das árvores a 1,30 $\mathrm{m}$ de altura do solo de cada desbaste, em $\mathrm{cm}$;

$\mathrm{H}$ - altura média das árvores de cada desbaste, em m;

FF - fator de forma, considerado 0,56 (Tonini et al., 2009);

$\mathrm{N}$ - número de árvores por hectare, calculado a partir da percentagem de desbaste.

Foi calculado o incremento médio anual (IMA) em volume, tomando-se a razão entre o volume do povoamento e sua idade (Loetsch et al., 1973).

A biomassa seca do fuste das árvores de desbastes foi estimada a partir da Equação 2, conforme concepções expostas por Ribeiro et al. (2009).

$$
B S d=V M d * D B
$$

Em que:

BSd - biomassa seca de madeira de desbastes, em kg.ha ${ }^{-1}$;

VMd - volume de madeira de desbaste médio, em $\mathrm{m}^{3} \cdot \mathrm{ha}^{-1}$;

DB - densidade básica da madeira de desbaste, em kg. $\mathrm{m}^{-3}$.

A densidade energética potencial de desbastes foi calculada considerando a produção de biomassa por hectare e o poder calorífico superior de cada desbaste (Equação 3), conforme Silva et al. (2014a).

$$
D E p=\left(B S d^{*} P C S\right) / 100
$$

Em que:

DEp - densidade energética potencial de madeira de desbastes, em kcal.ha-1;

BSd - biomassa seca de madeira de desbastes, em kg.ha ${ }^{-1}$;

PCS - poder calorífico superior da madeira de desbastes, em kcal.kg ${ }^{-1}$.

A estimativa da retirada de material mineral (inorgânico) do solo, devido à obtenção da madeira de desbastes, foi estimada com base no teor de cinzas, a partir da Equação 4, conforme concepções expostas por Schumacher \& Poggiani (1993).

$$
M M d=B S d * \frac{C Z}{100}
$$

Onde:

MMd $=$ material mineral presente na madeira de desbastes, em kg.ha-1;

$\mathrm{BSd}=$ biomassa seca de madeira de desbastes, em kg.ha' ${ }^{-1}$;

$\mathrm{CZ}=$ teor de cinzas médio na madeira de desbaste, em $\%$.

\section{Resultados e discussão}

Os resultados das variáveis dendrométricas: altura das árvores desbastadas, DAP, volume de madeira, biomassa seca e número de árvores de teca por hectare estão apresentados na Tabela 1.

Tabela 1. Médias das variáveis dendrométricas da madeira de Tectona grandis por desbaste, São José dos Quatro Marcos, MT.

\begin{tabular}{lcc}
\hline \multicolumn{1}{c}{ Variáveis dendrométricas } & $\mathbf{1}^{\mathbf{0}}$ desbaste & $\mathbf{2}^{\mathbf{0}}$ desbaste \\
\hline Árvores $\left(\mathrm{N}^{\mathrm{o}} \cdot \mathrm{ha}^{-1}\right)$ & 370 & 206 \\
Idade (meses) & 56 & 92 \\
Altura das árvores desbastadas (m) & 10,00 & 15,80 \\
Diâmetro à 1,30 m do solo (cm) & 11,50 & 18,30 \\
Incremento médio anual $\left(\mathrm{m}^{3} \cdot \mathrm{ha}^{-1}\right)$ & 10,30 & 20,50 \\
Volume de madeira $\left(\mathrm{m}^{3} \cdot \mathrm{ha}^{-1}\right)$ & 21,51 & 47,92 \\
Biomassa seca $\left(\mathrm{kg} \cdot \mathrm{ha}^{-1}\right)$ & $9.558,10$ & $27.794,80$ \\
\hline
\end{tabular}

Os maiores valores médios de altura e DAP, ocorreram no segundo desbaste do plantio de teca (tabela 1), resultando em maior produção de biomassa e volume total no desbaste aos 92 meses, sendo este quase três vezes maior que o desbaste realizado aos 56 meses.

Os valores médios encontrados para altura e DAP, no desbaste aos 56 meses, foram superiores aos observados por Garcia (2006) em um plantio de T. grandis, com 60 meses e espaçamento de $3 \mathrm{~m}$ x $3 \mathrm{~m}$, no Mato Grosso, cujo valores do DAP variaram entre $8,43 \mathrm{~m}$ e $9,87 \mathrm{~m}$ e a altura variou entre $6,60 \mathrm{~m}$ e $7,84 \mathrm{~m}$. Entretanto, foi inferior aos valores observados por Vieira et al. (2007), em um experimento de teca realizado em Rondônia, com espaçamento $3 \mathrm{~m} \mathrm{x} 4 \mathrm{~m}$, e por Pelissari et al (2013) em um plantio de teca, situado no estado do Mato Grosso, com espaçamento de $3 \mathrm{~m} \mathrm{x} 3 \mathrm{~m}$, ambos com 5 anos. Os valores do DAP e da altura observados no experimento em Rondônia foram 16,81 m e 14,20 m, respectivamente, e 13,76 m e 12,03 m no plantio de teca no Mato Grosso.

Os valores médios encontrados para DAP e altura, no desbaste aos 92 meses foram semelhantes aos observados 
por Kanninen et al. (2004) para a realidade do plantio de teca na Costa Rica e inferiores aos valores observados por Vieira et al. (2007) e Pelissari et al (2013), em que os valores do DAP e da altura foram $22,41 \mathrm{~m}$ e 20,15 $\mathrm{m}$, e $18,82 \mathrm{~m}$ e $16,96 \mathrm{~m}$, respectivamente, em plantios com 8 anos.

Os resultados estatísticos das propriedades dendroenergéticas estão apresentados na Tabela 2.

Tabela 2. Análise de variância das propriedades da madeira em função da posição ao longo do fuste e idades de desbaste de Tectona grandis, São José dos Quatro Marcos, MT.

\begin{tabular}{|c|c|c|c|c|c|c|}
\hline \multirow{2}{*}{$\begin{array}{l}\text { Fator de } \\
\text { variação }\end{array}$} & \multirow[t]{2}{*}{ GL } & \multirow{2}{*}{$\begin{array}{c}\text { DB } \\
\left(\mathrm{kg}^{-\mathrm{m}^{-3}}\right)\end{array}$} & \multirow{2}{*}{$\begin{array}{c}\text { PCS } \\
\left(\text { kcal.kg }^{-1}\right)\end{array}$} & MV & $\mathrm{CF}$ & TC \\
\hline & & & & \multicolumn{3}{|c|}{ (\%) } \\
\hline Posição & 2 & $0,00610^{\text {ns }}$ & $10337,79^{\mathrm{ns}}$ & $0,73085^{\text {ns }}$ & $0,84738^{\text {ns }}$ & $0,05828^{\mathrm{ns}}$ \\
\hline Desbaste & 1 & $0,13602 * *$ & $41897,00^{\mathrm{ns}}$ & $0,77748^{\text {ns }}$ & $0,52819^{\mathrm{ns}}$ & $0,01445^{\text {ns }}$ \\
\hline $\begin{array}{c}\text { Posição x } \\
\text { desbaste }\end{array}$ & 2 & $0,00032^{\mathrm{ns}}$ & $3039,01^{\text {ns }}$ & $0,04422^{\text {ns }}$ & $0,08122^{\mathrm{ns}}$ & $0,00337^{\mathrm{ns}}$ \\
\hline \multicolumn{2}{|c|}{$\begin{array}{c}\text { Coeficiente de variação } \\
(\%)\end{array}$} & 16,35 & 2,59 & 1,24 & 4,85 & 28,23 \\
\hline
\end{tabular}

$\mathrm{GL}=$ graus de liberdade; $\mathrm{DB}=$ densidade básica $\mathrm{PCS}=$ poder calorífico superior; $\mathrm{MV}=$ materiais voláteis; $\mathrm{CF}=$ carbono fixo; $\mathrm{TC}=$ teor de cinzas. **significativo a $1 \%$ pelo teste $\mathrm{F} ; \mathrm{ns}=$ não significativo a $5 \%$ pelo teste $\mathrm{F}$.

A análise de variância revelou não existir diferença significativa a $95 \%$ de probabilidade para o fator de variação "posição ao longo do fuste" em relação a todas as propriedades estudadas. Houve variação significativa a $99 \%$ de probabilidade somente para densidade básica em relação à idade de desbaste. O coeficiente de variação foi menor para as propriedades poder calorífico superior, teores de materiais voláteis e carbono fixo, e maiores para teor de cinzas e densidade básica.

Em relação ao poder calorífico superior no sentido axial, Silva et al. (2012) também não observaram variação significativa ao longo do fuste para as espécies Acacia mearnsii De Wild, Eucalyptus grandis W. Hill, Mimosa scabrella Benth. e Ateleia glazioveana Baill com um ano de idade. No entanto, Eloy et al. (2013; 2014a) observaram variações no sentido axial para a densidade básica da madeira para essas mesmas espécies com três anos de idade. Eloy et al. (2014a) observaram ainda que a idade do povoamento influenciou a densidade e o poder calorífico superior da madeira para as mesmas espécies.

O teor de materiais voláteis, teor de cinzas e carbono fixo não foram influenciados pela idade do povoamento ou pela variação axial. Essas tendências também foram observadas por Silva et al. (2014b) e Eloy et al. (2014a; 2014b) em trabalhos com diversas espécies para fins energéticos.
Na Tabela 3, estão apresentados os valores médios das propriedades dendroenergéticas das madeiras de desbaste.

Tabela 3. Médias das variáveis dendroenergéticas da madeira de Tectona grandis por desbaste, São José dos Quatro Marcos, MT.

\begin{tabular}{lcc}
\hline \multicolumn{1}{c}{$\begin{array}{c}\text { Variáveis } \\
\text { Dendroenergéticas }\end{array}$} & $\mathbf{1}^{\mathbf{0}}$ Desbaste & $\mathbf{2}^{\mathbf{0}}$ Desbaste \\
\hline $\begin{array}{l}\text { Densidade básica }\left(\mathrm{g} \cdot \mathrm{m}^{-3}\right) \\
\text { Poder calorífico superior } \\
\left.\text { (kcal.kg }{ }^{-1}\right)\end{array}$ & $0,442 \pm 0,04$ & $0,584 \pm 0,05$ \\
Materiais voláteis (\%) & $8.557 \pm 101$ & $4.632 \pm 114$ \\
Teor de carbono fixo (\%) & $18,52 \pm 1,04$ & $18,25 \pm 0,88$ \\
Teor de cinzas (\%) & $0,61 \pm 0,18$ & $0,57 \pm 0,17$ \\
$\begin{array}{l}\text { Densidade energética } \\
\text { (Mcal.ha- }\end{array}$ & $43557 \pm 968$ & $128746 \pm 3165$ \\
Material mineral $\left(\mathrm{Kg} \cdot \mathrm{ha}^{-1}\right)$ & $58,70 \pm 19,86$ & $158,49 \pm 70,89$ \\
\hline
\end{tabular}

A densidade básica média da madeira de $1^{\circ}$ desbaste (56 meses) foi de $0,442 \mathrm{~g} . \mathrm{cm}^{-3}$, inferior a do $2^{\circ}$ desbaste (92 meses) que foi $0,584 \mathrm{~g} . \mathrm{cm}^{-3}$. Isso ocorre, pois normalmente a densidade tende a aumentar com a idade (Vital et al., 1984). O valor para o $1^{\circ}$ desbaste está de acordo com o encontrado por Solorzano et al. (2012) para madeiras de teca com 4 anos. Para o $2^{\circ}$ desbaste, o resultado foi semelhante ao encontrado por Lima et al. 
(2009) para árvores de T. grandis provenientes de um experimento silvicultural situado no estado de São Paulo, com 31 anos e espaçamento $3 \mathrm{~m}$ x 2,5 m, e por Motta (2011) que encontrou uma densidade de $0,54 \mathrm{~cm} \cdot \mathrm{g}^{-3}$ para amostras de $T$. grandis com 15 anos provenientes de um plantio situado em Minas Gerais. Também foi semelhante ao encontrado por Cordero \& Kanninen (2003), Pérez \& Kanninen (2005) e Flórez (2012), cujos valores para árvores com 8 anos variaram entre 0,50 e 0,65 g. $\mathrm{cm}^{-3}$, em plantios de teca na Costa Rica.

As médias encontradas para poder calorífico superior no $1^{\circ}$ e $2^{\circ}$ desbastes $(4.557$ e 4.632 Kcal.kg-1 respectivamente) são estatisticamente iguais entre si, contudo, estudos demonstram que madeiras mais velhas podem apresentar maiores teores de extrativos, conforme foi determinado por (Silvério et al., 2006). O poder calorífico superior médio encontrado entre os desbastes e idades (4.595 Kcal. $\left.\mathrm{kg}^{-1}\right)$, foi inferior ao encontrado por Günther et al. (2012) para a madeira de teca (4.848 Kcal.kg-1), e semelhante ao encontrado por Santos (2010) para diferentes clones de eucalipto, com 7 anos e espaçamento $3 \mathrm{~m} \times 3 \mathrm{~m}$, que variou entre 4.274 e 4.585 Kcal. $\mathrm{kg}^{-1}$ e ao valor de $4.630 \mathrm{Kcal} \mathrm{kg}^{-1}$ para madeira de Eucalyptus pellita F. Muell com 5 anos (Oliveira et al., 2010).

Para análise química imediata da madeira de desbaste de $T$. grandis, pode-se utilizar como produto deste trabalho as médias entre as posições ao longo do fuste e as idades de desbaste: $80,37 \%$ materiais voláteis; $18,39 \%$ para carbono fixo; $0,59 \%$ para o teor de cinzas, tendo em vista que não houve variação em função da posição ao longo do fuste, idades de desbaste e nenhuma tendência na estatística descritiva dos resultados.

$\mathrm{O}$ teor de carbono fixo na madeira de $T$. grandis apresentou maiores valores, quando comparados com diferentes espécies de eucalipto (Brito \& Barrichelo, 1978), o que caracteriza uma madeira melhor para queima. Entretanto, o teor de cinza, foi superior ao encontrado pelos autores citados, o que não a desqualifica para a utilização como fonte de energia, uma vez que estando abaixo de $1 \%$ ela se encontra dentro dos padrões aceitáveis para a biomassa do tipo madeira. Os valores de 0,61 e $0,57 \%$ estão abaixo do teor de cinzas $(2,81 \%)$ encontrado para a madeira de teca proveniente de plantações na Costa Rica (Moya \& Tenório, 2013).

A densidade energética potencial calculada foi de 43.557 e 128.746 Mcal.ha $^{-1}$, respectivamente, para madeira de primeiro e segundo desbaste. $\mathrm{O}$ valor do segundo desbaste foi três vezes maior que o valor calculado para o primeiro, uma vez que a mesma relação foi encontrada para o volume total de biomassa de cada desbaste e a variável é diretamente proporcional à densidade energética calculada. $\mathrm{O}$ poder calorífico pouco influenciou este resultado, pois não apresentou diferença significativa entre os valores comparados. Os valores obtidos encontram-se bem abaixo dos valores encontrados para plantações de eucaliptos com características de espaçamento e idade semelhantes (Santos, 2010; Santos et al., 2012) isto porque o incremento médio anual da teca é menor do que o do eucalipto.

Com base nas variáveis dendrométricas e dendroenergéticas determinadas, foi possível estimar a quantidade de material mineral (inorgânico) presente na madeira de desbaste de teca. Foi observada na madeira de segundo desbaste, uma quantidade de material mineral superior ao do primeiro desbaste (Tabela 3). Embora o teor de cinzas (material mineral) ter sido semelhante nos materiais dos dois desbastes, a produção maior de biomassa seca de teca (Tabela 1) explica a diferença da quantidade de material mineral entre as madeiras dos desbastes.

Conhecer a quantidade e a composição do material mineral de biomassa para uso energético é importante, pois fazem parte do vegetal e, sendo assim, participam dos processos de obtenção de energia como material inerte. Andrade et al. (1999) e Poggiani (1980), afirmam que o lenho de desbaste possui menor porcentagem da fração mineral em relação à fração orgânica do vegetal, mas deve ser considerado para manter o nível de ciclagem de nutrientes e a quantidade no solo para fins florestais. Baggio \& Carpanezzi (1997), afirmam que o ideal é conhecer o quantitativo da fração mineral, bem como sua composição.

\section{Conclusões}

Não há variação significativa para as propriedades: densidade básica, poder calorífico superior, materiais voláteis, carbono fixo e teor de cinzas em função das posições ao longo do fuste: base, meio e topo e da idade do povoamento de Tectona grandis.

Não foi observada diferença significativa nas propriedades dendroenergéticas entre as madeiras de desbaste, exceção para densidade básica que foi maior para madeira de segundo desbaste de Tectona grandis. 


\section{Referências}

ANDRADE, A. G. de; CABALLERO, S. S. U.; FARIA, S. M. de. Ciclagem de nutrientes em ecossistemas florestais. Rio de Janeiro: Embrapa Solos, 1999. 50 p. (Embrapa Solos. Documentos, 13). Disponível em: <http://www.infoteca.cnptia.embrapa.br/handle/ doc/337399>. Acesso em: 22 maio 2015.

ANGELI, A. Tectona grandis (Teca). In: IDENTIFICAÇÃO de espécies florestais. Piracicaba: IPEF, 2003. Disponível em: $<$ http:// www.ipef.br/identificacao/tectona. grandis.asp $>$. Acesso em: 17 set. 2015.

ASSOCIAÇÃO BRASILEIRA DE NORMAS TÉCNICAS. NBR 8112: carvão vegetal: análise imediata: método de ensaio. Rio de Janeiro, 1986. 5 p.

ASSOCIAÇÃO BRASILEIRA DE NORMAS TÉCNICAS. NBR 8633: carvão vegetal: determinação do poder calorífico. Rio de Janeiro, 1984. 13 p.

ASSOCIAÇÃO BRASILEIRA DE NORMAS TÉCNICAS. NBR 11941: madeira: determinação da densidade básica. Rio de Janeiro, 2003a. 6 p.

BAGGIO, A. J.; CARPANEZZI, A. A. Estoque de nutrientes nos resíduos da exploração de bracatingais. Boletim de Pesquisa Florestal, Colombo, n. 34, p. 17-29, jan./jun., 1997. Disponível em: $<$ http://www.alice.cnptia.embrapa.br/handle/doc/282162>. Acesso em: 05 jul. 2014.

BRITO, J. O.; BARRICHELO, L. E. G. Características do eucalipto como combustível: análise química imediata da madeira e da casca. IPEF, Piracicaba, n. 16, p. 63-70, 1978. Disponível em: <http:// www.ipef.br/publicacoes/scientia/nr16/cap05.pdf >. Acesso em: 03 de jul. de 2014.

CORDERO, L. D. P.; KANNINEN, M. Heartwood, sapwood and bark content, and wood dry density of young and mature teak (Tectona grandis) trees grown in Costa Rica. Silva Fennica, Helsinki, v. 37 , n. 1, p. $45-54.2003$.

ELOY, E.; CARON, B. O.; SILVA, D. A.; SCHMIDT, D.; TREVISAN, R.; BEHLING, A.; ELLI, E. F. Influência do espaçamento nas características de espécies arbóreas em plantios de curta rotação. Revista Árvore, Viçosa, MG, v. 38, n. 3, p. 551-119, 2014a. DOI: 10.1590/S0100-67622014000300018.

ELOY, E. CARON, B. O.; TREVISAN, R.; BEHLING, A.; SOUZA, V.Q.; SCHMIDT, D. Variação axial e efeito do espaçamento na massa específica da madeira de Eucalyptus grandis e Acacia mesrnsii. Ciência da Madeira, v. 4, n. 2, p. 215-227, 2013. DOI: 10.12953/2177-6830.v04n02a07

ELOY, E.; SILVA, D. A.; CARON, B. O.; SOUZA, V. Q.; BEHLING, A.; ELLI, E. F.; MONTEIRO, G. C. Caracterização da biomassa da madeira e da casca de Mimosa scrabrella Benth cultivada em dois diferentes espaçamentos. Ciência da Madeira, v. 6, n.1, p. 38-46, 2014b. DOI: 10.12953/2177-6830/rcm.v6n1p38-46.

FLÓREZ, B. J. Caracterização tecnológica da madeira jovem de teca (Tectona grandis L.f). 2012. 85 f. Dissertação (Mestre em Ciência e Tecnologia da madeira) - Universidade Federal de Lavras. Lavras.
GARCIA, M. L. Intensidade de desbaste em um povoamento de Tectona grandis L.f., no município de Sinop- MT. 2006. 44 f. Dissertação (Mestre em Agricultura Tropical) - Faculdade de Agronomia e Medicina Veterinária, Universidade Federal de Mato Grosso, Cuiabá.

GONZÁLEZ, W. F. Manual para productores de teca (Tectona grandis L. f) en Costa Rica. Heredia: Costa Rica, 2004. 121 p.

GÜNTHER, B.; GEBAUER, K.; BARKOWSKI, R.; ROSENTHAL M.; BUES, C.-T. Calorific value of selected wood species and wood products. European Journal of Wood and Wood Products, v. 70, n. 5, p. 755-757, May. 2012. DOI 10.1007/s00107-012-0613-z

KANNINEN, M.; CORDERO, L. D. P.; MONTERO, M.; VÍQUEZ, E. Intensity and timing of the first thinning of Tectona grandis plantations in Costa Rica: results of a thinning trial. Forest Ecology and Management, Amsterdam, n. 203, p. 89-99, 2004. DOI: 10.1016/j.foreco.2004.07.054

LIMA, I. L.; FLORSHEIM, S. M. B.; LONGUI, E. L. Influência do espaçamento em algumas propriedades físicas da madeira de Tectona grandis Linn. Cerne, Lavras, v. 15, n. 2, p. 244-250, abr.jun, 2009.

LOETSCH, P.; ZÖHRER, P.; HALLER, K.E. Forest inventory. Munich: BLV, 1973. v. 2. 469 p.

MOTTA, J. P. Propriedades tecnológicas da madeira de Tectona grandis L.f. proveniente do Vale do Rio Doce, Minas Gerais. 2011. 125 f. Dissertação (Mestre em Ciências Florestais) -Universidade Federal do Espírito Santo, Jerônimo Monteiro.

MOYA, R.; TENORIO, C. Fuelwood characteristics and its relation with extractives and chemical properties of ten fast-growth species in Costa Rica. Biomass and Bioenergy, Oxford, v. 56, p. 14-21, Set. 2013. DOI: $10.1016 /$ j.biombioe.2013.04.013

NOCETTI, M.; ROZENBERG, P.; CHAIX, G.; MACCHIONI, N. Provenance effect on the ring structure of teak (Tectona grandis L.f.) wood by X-ray icrodensitometry. Annals of Forest Science, Les Ulis, v. 68, p. 1375-1383, 2011. DOI: 10.1007/s13595-011-0145-4.

OLIVEIRA, A. C.; CARNEIRO, A. de C. O.; VITAL, B. R.; ALMEIDA, W.; PEREIRA, B. L. C.; CARDOSO, M. T. Parâmetros de qualidade da madeira e do carvão vegetal de Eucalyptus pellita F. Muell. Revista Scientia Forestalis, Piracicaba, v. 38, n. 87, p. 431-439, 2010

PANDEY, D.; BROWN, C. Teak: a global overview. Unasylva, v. 51, n. 201, p. 3-13, 2000.

PELISSARI, A. L.; CALDEIRA, S. F.; DRESCHER R. Desenvolvimento Quantitativo e Qualitativo de Tectona grandis L.f. em Mato Grosso. Floresta e Ambiente, Seropédica, v. 20, n. 3, p. 371-383, jul./set. 2013. DOI: 10.4322/floram.2013.027

PELISSARI, A. L.; GUIMARÃES, P. P.; BEHLING, A.; EBLING, A. A. Cultivo da teca: características da espécie para implantação e condução de povoamentos florestais. Agrarian Academy, v. 1, n. 1, p. $127-145,2014$

PÉREZ, D.; KANNINEN, M. Effect of thinning on stem form and wood characteristics of teak (Tectona grandis) in a humid tropical site in Costa Rica. Silva Fennica, Helsinki, v. 39, n. 2, p. 217-225, 2005

POGGIANI, F. Florestas para fins energéticos e ciclagem de nutrientes. Série Técnica IPEF, Piracicaba, v. 1, n. 2, p. D.1-D.11, Jul.1980. 
RIBEIRO, S. C.; JACOVINE, L. A. G.; SOARES, C. P. B; MARTINS, S. V; SOUZA, A. L.; NARDELLI, A. M. B. Quantificação de biomassa e estimativa de estoque de carbono em uma floresta madura no município de Viçosa, Minas Gerais. Revista Àrvore, Viçosa, MG, v. 33, n. 5, p. 917-926. Sept./Out. 2009. DOI: 10.1590/S0100-67622009000500014.

SANTOS, L. C.; CARVAlHO, A. M. M. L.; PEREIRA, B. L. C.; OlIVEIRA, A. C.; CARNEIRO, C. O.; TRUGILHO, P. F. Propriedades da madeira e estimativas de massa, carbono e energia de clones de Eucalyptus plantados em diferentes locais. Revista Árvore, Viçosa, MG, v. 36, n. 5, out. 2012. DOI: 10.1590/S010067622012000500019 .

SANTOS, R. C. Parâmetros de qualidade da madeira e do carvão vegetal de clones de eucalipto. 2010. 173 f. Tese (Doutorado em Ciência e Tecnologia da Madeira) - Universidade Federal de Lavras, Lavras.

SCHUHLI, G. S.; PALUDZYSZYN FILHO, E. O cenário da silvicultura de teca e perspectivas para o melhoramento genético. Pesquisa Florestal Brasileira, Colombo, v. 30, n. 63, p. 217-230, 2010. DOI: 10.4336/2010.pfb.30.63.217.

SCHUMACHER, M. V.; POGGIANI, F. Produção de biomassa e remoção de nutrientes em povoamentos de Eucalyptus camaldulensis Dehnh., Eucalyptus grandis ex Maiden e Eucalyptus torelliana F. Muell, plantados em Anhembi, SP. Ciência Florestal, Santa Maria, RS, v. 3, n. 1, p. 21-34, dez. 1993.

SHIMIZU, J. Y.; KLEIN, H.; OLIVEIRA, J. R. V. Diagnóstico das plantações florestais em Mato Grosso. Cuiabá: Central de Texto, 2007. 63 p.

SILVA, D. A.; ALMEIDA, V. C.; VIANA, L. C.; KLOCK, U.; MUÑIZ, G. I. B. Avaliação das propriedades energéticas de resíduos de madeiras tropicais com uso da espectroscopia NIR. Floresta e Ambiente, Seropédica, v. 21, n. 4, p. 561-568, 2014a. DOI: 10.1590/2179-8087.043414.

SILVA, D. A. da. Avaliação da eficiência energética em uma indústria de painéis compensado. 2001. 205 f. Tese (Doutorado em Ciências Florestais) -Universidade Federal do Paraná, Curitiba.

SILVA, D. A.; CARON, B. O.; BEHLING, A.; SOUZA, V. Q.; ELOY, E. Ponto de amostragem ao longo do fuste para estimativa do poder calorífico da madeira. Ciência Rural, Santa Maria, RS, v. 42, n. 9, p. 1588-1595, 2012. DOI: 10.1590/S0103-84782012000900012.
SILVA, D. A.; CARON, B. O.; SANQUETTA, C. R.; BEHLING, A.; SCHMIDT, D.; BAMBERG, R.; ELOY, E.; CORTE, A. P. D. Equations for estimating gross calorific value of wood from four tree species. Revista Chapingo Serie Ciencias Forestales y del Ambiente, v. 20, n. 2, p. 177-186, abr. 2014b. DOI: 10.5154/r. rchscfa. 2013.09.035.

SILVA, D. A.; JASKIU, E.; RIBEIRO, R. M.; MARCHIORI, F.; MAYER, S. L. S; BRANDELICK, C. C. M. Participação da energia de base florestal na matriz energética brasileira. In: ENCONTRO BRASILEIRO EM MADEIRAS E EM ESTRUTURAS DE MADEIRA, 12., 2010, Lavras. Madeira para todos: os desafios: anais. Lavras: UFLA, 2010. CD-ROM.

SILVÉRIO, F. O.; BARBOSA, L. C. de A.; GOMIDE, J. L.; REIS, F. P.; PILÓ-VELOSO, D. Metodologia de extração e determinação do teor de extrativos em madeiras de eucalipto. Revista Árvore, Viçosa, MG, v. 30, n. 6, p. 1009-1016, 2006.

SOLORZANO, S.; MOYA, R; MURILLO, O. Early prediction of basic density, shrinking, presence of growth stress, and dynamic elastic modulus based on the morphological tree parameters of Tectona grandis. Journal of Wood Science, v. 58, n. 4, p. 290-299, Aug. 2012. DOI: 10.1007/s10086-012-1261-y.

TONInI, H.; COSTA, M. C. G.; SCWEnGBER, L. A. M. Crescimento de Teca (Tectona grandis) em reflorestamento na Amazônia Setentrional. Pesquisa Florestal Brasileira, Colombo, v. 4, n. 59, p. 5-14, 2009. DOI: 10.4336/2009.pfb.59.05.

TROPICOS. Missouri botanical garden. 2015. Disponível em: $<$ http://www.tropicos.org>. Acesso em: 15 set. 2015.

VIEIRA, A. H.; ROCHA, R. B.; LOCATELLI, M.; GAMA, M. de M. B.; TEIXEIRA, C. A. D.; MARCOLAN, A. L.; JUNIOR, J. R. V. Sistema de produção de teca para o Estado de Rondônia. Porto Velho: Embrapa Rondônia, 2007. 18 p. (Embrapa Rondônia. Sistemas de Produção, 30). Disponível em: <http://www.infoteca. cnptia.embrapa.br/bitstream/doc/698944/1/spteca.pdf $>$. Acesso em: 20 jun. 2014.

VITAL, B. R.; PEREIRA, A. R.; DELLA-LUCIA, R. M.; ANDRADE, D. C. Efeito da idade da árvore na densidade da madeira de Eucalyptus grandis cultivado na região do cerrado de Minas Gerais. Revista Brasil Florestal, Brasília, DF, n. 8, p. 49-52, 1984. 
